\title{
Time-resolved high-resolution spectroscopy of the bright pulsating subdwarf B star Balloon 090100001*
}

\author{
J. H. Telting ${ }^{1}$, S. Geier ${ }^{2}$, R. H. Østensen ${ }^{3}$, U. Heber ${ }^{2}$, L. Glowienka ${ }^{1}$, T. Nielsen ${ }^{1}$, R. Oreiro ${ }^{3}$, and S. Frandsen ${ }^{4}$ \\ 1 Nordic Optical Telescope, Apartado 474, 38700 Santa Cruz de La Palma, Spain \\ e-mail: jht@not.iac.es \\ 2 Dr. Remeis Sternwarte Bamberg, Universität Erlangen-Nürnberg, Germany \\ ${ }^{3}$ Instituut voor Sterrenkunde, Katholieke Universiteit Leuven, Belgium \\ 4 Department of Physics and Astronomy, Århus University, Denmark
}

Received 6 August 2008 / Accepted 23 October 2008

\section{ABSTRACT}

\begin{abstract}
We have obtained, for the first time, a time-resolved high-resolution spectroscopic data set of the bright sdBV Balloon 090100001. In the autumn of 2006 we gathered over 1600 time-resolved high-resolution spectra with the aim to identify the pulsation modes in Balloon 090100001 by studying the line-profile variations. In this paper we present the results obtained from 842 spectra secured in August 2006, aiming to identify the dominant pulsation mode in this star.

We phase fold the spectra onto the known dominant period of $2.80746 \mathrm{mHz}$, to study the abundances and line-broadening as a function of pulsation phase. We find that the abundances do not stand out against those of other subdwarf B stars. Consequently, there is no way to demonstrate that the richness of the frequency spectrum and/or the high pulsation amplitudes are related to abundance effects. The abundances do not change with pulsational phase.

The metal lines in Balloon 090100001 are much broader than for non-pulsating subdwarfs, and during the cycle of the main pulsation the line broadening varies with $\sim 2.5 \mathrm{~km} \mathrm{~s}^{-1}$ amplitude. Hence, we find clear evidence of pulsational broadening of the lines.

We combine the information content of 56 narrow absorption lines through a cross-correlation technique, to create cross-correlation functions that for each individual pulsation phase approximate the average line-profile shape. The resulting profiles have a sufficient signal-to-noise ratio for a mode-identification analysis. From the cross-correlation analysis we find that the pulsation amplitude of the main mode decreased from $19 \mathrm{~km} \mathrm{~s}^{-1}$ in 2004 to $14.5 \mathrm{~km} \mathrm{~s}^{-1}$ in 2006.

We present, for an sdB star, the first application of pulsation-mode identification based on line-profile variations of lines of heavy elements. To fit the line profiles we consider all modes with degree $\ell \leq 2$ and associated azimuthal order $m$, and we use a model of the pulsational surface-velocity field. The models with $\ell=2$ do not fit the profiles well, and consequently we can rule out quadrupole modes as the origin of the main pulsation mode in Balloon 090100001. Best fits are obtained from the model of a radial pulsation $(\ell=0)$ and the model of a dipole pulsation $(\ell=1)$.
\end{abstract}

Key words. stars: subdwarfs - line: profiles - stars: early-type - stars: oscillations - stars: variables: general stars: individual: Balloon 090100001

\section{Introduction}

Subdwarf B (sdB) stars collectively form the cooler of the populations on the hot extreme horizontal branch (EHB). They are core helium burning objects with an extremely thin $\left(M_{\text {env }} \lesssim 0.02 M_{\odot}\right)$ hydrogen-dominated envelope (Heber 1986; Saffer et al. 1994). An sdB star evolves towards the hotter sdO EHB population before reaching degeneracy and the associated white-dwarf cooling track (Dorman et al. 1993).

Although the future evolution of the $\mathrm{sdB}$ stars is generally accepted, their past formation into the sdB stage is under debate. Several scenarios that involve either single-star or binary evolution were proposed. The main single-star scenario requires enhanced mass loss during or after the red-giant branch phase (D'Cruz et al. 1996). The question in this case is how the massloss mechanism removes all but a tiny fraction of the hydrogen envelope at the same time as the He core has attained the mass $\left(\sim 0.5 M_{\odot}\right)$ required for the He flash. Several binary evolution scenarios have been proposed to explain the high number of detected binary sdB stars. Such scenarios involve close-binary

* Based on observations from the Nordic Optical Telescope. evolution with strong mass transfer in the form of either a common-envelope ejection, stable Roche-lobe overflow or a complete merger of two helium white dwarfs (see e.g. Han et al. 2002, 2003).

A theoretical prediction of the existence of pulsations in $\mathrm{sdB}$ stars, due to an opacity bump associated with iron ionisation in subphotospheric layers, was made by Charpinet (1996, 1997). Since both $p$ and $g$-mode pulsations were discovered in sdB stars (Kilkenny et al. 1997; Green et al. 2003), there has been a focus on the possibilities to derive the internal structure and to put constraints on the lesser known stages of the evolution by means of asteroseismology. Currently the immediate aims of asteroseismology of subdwarf B stars are to derive the mass of the helium-burning core and the mass of the thin hydrogen envelope around the core (e.g. Randall et al. 2006).

Due to its convenient brightness $(B=11.8)$ and very high pulsation amplitudes, the sdB star Balloon 090100001 has been intensely observed photometrically, leading to the recent discovery of more than 50 detected pulsational frequencies (Oreiro et al. 2004, 2005; Baran et al. 2006). Amongst these are $p$ - and $g$-mode frequencies, including a triplet close to 
the dominant frequency of $2.8075 \mathrm{mHz}$ (i.e. the pulsation with period of $356.2 \mathrm{~s}$ ). Altogether, the brightness, the pulsational amplitude, and the rich but seemingly non-randomly distributed frequency spectrum, make this star an excellent target for asteroseismological studies.

For asteroseismology the observations reveal the eigenvalues (pulsation frequencies), and the tangential part of the eigenfunctions (pulsation modes), as well as basic stellar parameters such as the surface temperature and gravity. Although it is possible to attempt a seismological analysis based on frequencies only, the results can in some cases be relatively unconstrained. More conclusive results can be obtained if the pulsation modes can be determined and identified observationally, as suggested by e.g. Charpinet et al. (2005, PG1219).

Very recently Van Grootel et al. (2008) showed that for Balloon 090100001 the asteroseismic results derived so far, i.e. total stellar mass and envelope mass, at the typical accuracy presently achieved do not depend much on the exact value of observationally determined values of the pulsational degree $\ell$. However, we anticipate that, as for many other types of pulsating stars, many stellar-interior parameters of sdB stars will preferrably be estimated through seismic studies based on observationally constrained identifications of $\ell$ and the azimuthal number $m$. This will become particularly important as asteroseismic models improve in sophistication and precision to the point where differences in internal structure caused by differing evolutionary channels can actually be tested, as demonstrated recently by Hu et al. (2008).

Observational mode identifications can be obtained from two complementary techniques. Firstly, there is the technique of photometric/spectroscopic amplitude ratios (e.g. Jeffery et al. 2004), which probes the surface temperature and gravity, and optionally also uses the radial-velocity amplitude as an extra constraint (e.g. Baran et al. 2008, for the case of Balloon 090100001). With this technique, that requires non-adiabatic modeling of the pulsations, one can derive the degree $\ell$ of the mode.

In this paper we present, for $\mathrm{sdB}$ stars, the first attempt to use a different method of mode identification that probes the 3D velocity field on the stellar surface by studying the pulsational line-profile variations in time-resolved high-spectral-resolution spectra. With this method, one can derive both the modal degree $\ell$ and the azimuthal number $m$; the latter is crucial for transforming the observed frequencies to those in the corotating frame of the star. We apply this method to our new high-resolution spectra of the sdB star Balloon 090100001 in order to identify the main pulsation mode.

Examples of time-resolved low-resolution spectroscopy of sdB stars are given for e.g. PB 8783 and KPD 1209+4401 (Jeffery \& Pollacco 2000), PG1605+072 (O'Toole et al. 2000, 2003), and PG1325+101 (Telting \& Østensen 2004). Recent high-resolution FUSE time-series of three sdBV were presented by Kuassivi et al. (2005). The only other time-resolved highspectral-resolution study of a pulsating sdB star presented in the literature so far is that of NY Vir (Vučković et al. 2007).

The mode-identification methods that essentially fit pulsational line-profile variations are well established for mainsequence stars (e.g. Briquet \& Aerts 2003; Telting \& Schrijvers 1997) but rely on high spectral resolution and high signal-tonoise line profiles. Given the short pulsation period, hence short exposure times, and the relative faintness of the subdwarf stars, it was generally thought that the application of such methods was out of reach for the subdwarf pulsators. In this paper we show that by using phase folding (e.g. Telting \& Østensen 2004; Kuassivi et al. 2005) and a subsequent cross-correlation
Table 1. Log of the high-resolution spectra that we discuss in this paper, obtained with the FIES spectrograph at the NOT. Dates are for start of night. HJD is given in seconds with respect to 2453952.0.

\begin{tabular}{cccc}
\hline \hline Date 2006 & \multicolumn{2}{c}{ Heliocentric JD } & Number of spectra \\
\hline 3 Aug. & -42782 & -21936 & 385 \\
4 Aug. & 43871 & 64754 & 457 \\
\hline
\end{tabular}

technique (e.g. Woolf et al. 2002) we can combine the pulsational information content of the individual lines and of the individual spectra in order to obtain profiles of sufficient quality for a detailed variability analysis. Our application of this method serves as an independent observational mode identification of the main mode in Balloon 090100001, to be compared to the results of other mode-ID techniques for this star (Baran et al. 2008; Charpinet et al. 2008).

Additionally, we use our new high-resolution spectra of Balloon 090100001 to do a detailed abundance and linebroadening analysis. We investigate the origin of the line broadening in pulsating subdwarfs, and check whether the high pulsation amplitudes in this star may be related to high metallicity.

The analysis and modelling of our low-resolution timeresolved spectroscopy of Balloon 090100001 can be found in Østensen et al. (2008) and Telting et al. (2006). These data have resulted in characterisation of the pulsational surface temperature and gravity variations, and in a first conclusive modeidentification of the dominant pulsation in Balloon 090100001 (Baran et al. 2008). In this paper we present the first results from time-resolved high-resolution spectroscopy of Balloon 090100001, based on a new data set that was obtained with the aim to put further constraints on the pulsational characteristics of this star. Preliminary results were presented in Telting et al. (2008). Here we focus on the identification of the dominant pulsation mode in Balloon 090100001; our data set will be scrutinised to investigate the information content of the smalleramplitude pulsations in a separate paper.

\section{Observations}

On altogether 6 nights spread out over Aug.-Dec. 2006 we obtained new time-resolved high-resolution spectra of Balloon 090100001 using the $2.6 \mathrm{~m}$ Nordic Optical Telescope (NOT) with the FIES Echelle spectrograph with its lowestresolution fiber. More than half the total number of spectra were obtained on two consecutive nights in Aug. 2006 (see the observing $\log$ in Table 1). To minimise the overheads we used $3 \times 2$ (spatial $\times$ spectral) binning and the fast dual-amplifier read mode. The setup gave a resolution of $R=25000$ (Gaussian $\sigma=5 \mathrm{~km} \mathrm{~s}^{-1}$ ), and with $30 \mathrm{~s}$ exposure time our cycle time was $43 \mathrm{~s}$. Note that the dual-amplifier read mode caused that the central Echelle order is lost, implying that the wavelength region 4487-4502 A was not recorded in our spectra.

In this paper we present results based on the spectra obtained in August 2006 only, aiming to identify the pulsation mode of the dominant pulsation frequency. The use of only the August 2006 data implies that we do not need to know the pulsation frequency to highest accuracy in order to phase-fold the data (see next subsection). In a later paper we will analyse our full data set, including a full Fourier analysis, to investigate its information content for the lower-amplitude pulsations in this star. 


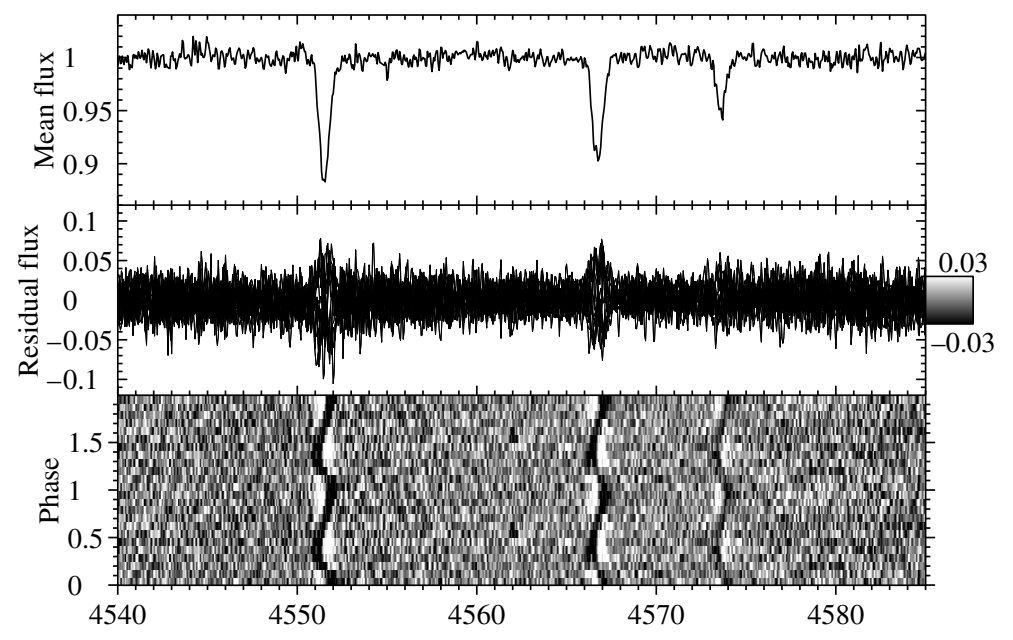

Fig. 1. Time-resolved high-resolution spectroscopy of Balloon 090100001 after binning the 842 spectra of August 2006 according to the phase of the main pulsation mode. We here show the spectral region of the SilII triplet: mean spectrum (top), and mean-subtracted phase-binned spectra (middle and lower panels). The grey-scale bar indicates the cut levels used for the bottom plot.

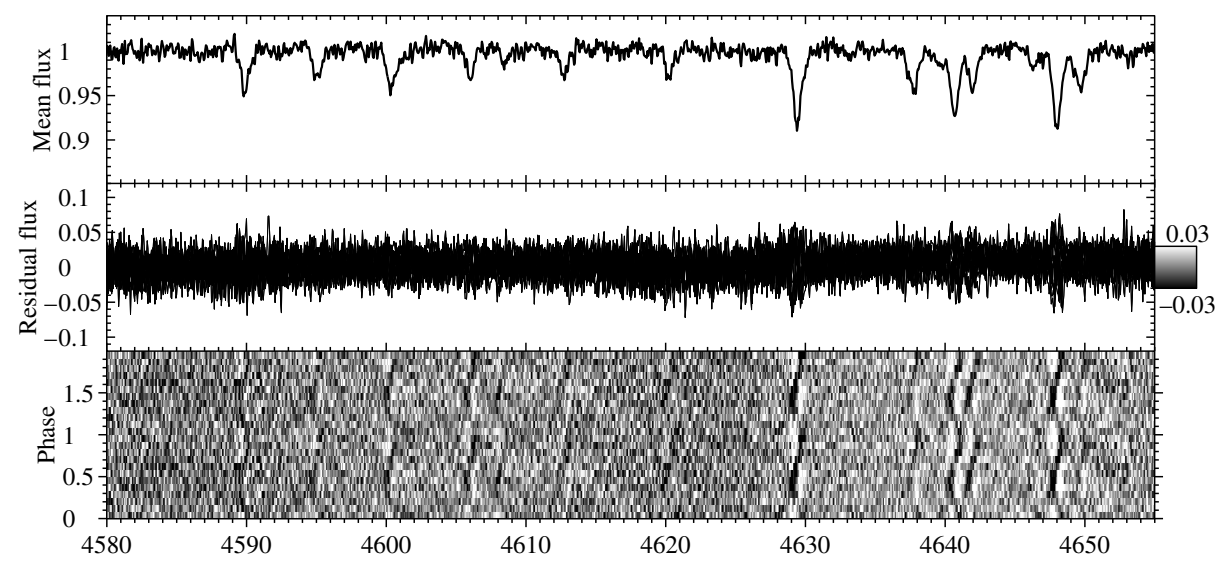

Fig. 2. As Fig. 1, showing many variable lines of OII and NII.

\subsection{Reduction and phase folding}

For pulsation-mode identification one needs high-resolution spectra with high $S / N$. Due to the short pulsation periods (for the dominant mode $356.2 \mathrm{~s}$ ), and the relative faintness of the star $(B \sim 11.8)$, the $S / N$ for the individual spectra is very low. Therefore, we can only reach our objective by folding the individual spectra to pulsational phase.

We used standard IRAF routines to reduce the spectra. Rather than reducing all low- $S / N$ spectra individually we chose for a different approach: after scattered-light subtraction we phase folded the individual full-size CCD frames onto the known pulsation frequency of the main mode $2.80746 \mathrm{mHz}$ (e.g. Baran et al. 2008), into 12 bins of pulsational phase $\phi$, with $\phi=0$ corresponding to $\mathrm{HJD}=2453952.0$. For each of the 12 phase bins the CCD frames were combined while giving weights proportional to the inverse of the variance of the individual spectra. In the combining process, after scaling, the 3 highest and 2 lowest count values were rejected before averaging; cosmics were clipped in this process. As the main-mode frequency was chosen for the phase binning, the line-profile variability caused by modes other than the main mode was, to a large extent, averaged out. As the contrast between the amplitudes of the dominant mode and all other modes is high, we do not expect a significant contribution of the other modes to the variations in the remaining phase-folded spectra.
This left us with only 12 phase-binned full-size CCD frames to extract the Echelle spectra from. After variance-weighted spectral extraction the resulting peak $S / N$ was $\sim 50$ for each phase-binned spectrum. The peak signal-to-noise of the individual (not phase-binned) spectra was at most $S / N=10$. For the average spectrum obtained from all 842 spectra taken in August 2006 we obtained peak $S / N \sim 180$. Wavelength calibration was obtained from a ThAr lamp.

Due to the fact that the width of a single Balmer line spans about two full Echelle orders, and the inherent difficulties this poses on spectral normalisation, we could not attempt to accurately normalise the spectral regions near Balmer-line cores. This implies that we cannot derive temperature/gravity variations that are based on spectral fits of the Balmer line (wings), as can be done with low-resolution spectra, but note that this has already been done in the case of Balloon 090100001 by Telting et al. (2006) and Østensen et al. (2008). Additionally, our abundance analysis has to be restricted to helium/metal lines that are well away from the Balmer-line cores, because of the effect that Balmer-line opacity has on the depth of the helium/metal lines. Outside the Balmer-line cores our normalisation is adequate to study abundance and line-profile variations as a function of pulsational phase. Parts of the phase-binned and the average spectra are shown in Figs. 1 and 2. 
Table 2. Elemental abundances of Balloon 090100001. Average values with standard deviations are given, if more than one line was fitted. Single line measurements are given without errors. Upper limits are only given, if they constrain the abundances in a reasonable way. $\log \epsilon=12-\log (n(M) / n(H)) .[M / H]$ abundances relative to solar values are taken from Grevesse \& Sauval (1998). The abundances of the comparison star Feige 48 are derived from optical spectra using mostly the same line data as well as line lists, and are taken from Heber et al. (2000).

\begin{tabular}{lllll}
\hline \hline & & & & Feige 48 \\
Ion & $N_{\text {lines }}$ & $\log \epsilon$ & {$[M / H]$} & $\log \epsilon$ \\
\hline C II & 2 & $6.60 \pm 0.14$ & -1.92 & \\
C III & 1 & 7.10 & -1.42 & $7.36 \pm 0.03$ \\
N II & 11 & $7.55 \pm 0.12$ & -0.37 & $7.47 \pm 0.17$ \\
N III & & $<7.90$ & $<-0.02$ & $7.80 \pm 0.11$ \\
O II & 10 & $7.78 \pm 0.08$ & -1.05 & $7.79 \pm 0.12$ \\
Mg II & 1 & 6.80 & -0.78 & 6.91 \\
Al III & & $<5.66$ & $<-0.81$ & $5.50 \pm 0.18$ \\
Si III & 3 & $6.83 \pm 0.15$ & -0.72 & $6.39 \pm 0.32$ \\
Si IV & & $<6.98$ & $<-0.57$ & 6.17 \\
P III & & $<4.94$ & $<-0.51$ & \\
S II & & $<6.50$ & $<-0.83$ & \\
S III & 1 & 6.60 & -0.73 & 6.15 \\
Ar II & & $<6.80$ & $<0.40$ & \\
Ti III & & $<5.68$ & $<0.66$ & \\
Fe III & 8 & $7.38 \pm 0.23$ & -0.12 & $7.55 \pm 0.19$ \\
\hline
\end{tabular}

\section{Abundance and line-broadening analysis}

We have done a detailed abundance and line-broadening analysis aiming to detect possible variations as a function of the pulsational phase of the main mode. In order to derive the metal abundances we compared the observed spectra with synthetic line profiles. Metal line-blanketed LTE model atmospheres were computed for the stellar parameters $T_{\text {eff }}=28700 \mathrm{~K}$, $\log g=5.39$ and $\log (n(\mathrm{He}) / n(\mathrm{H}))=-2.70$ derived from low resolution spectra and given in Østensen et al. (2008) using the LINFOR program (developed by Holweger, Steffen and Steenbock at Kiel university, modified by Lemke 1997; see Heber et al. 2000; O'Toole \& Heber 2006, for details). We selected a standard set of 183 metal lines from 24 different ions and used atomic data from the list of Kurucz (1992). For carbon, nitrogen, oxygen and silicon, the NIST database was used to obtain state of the art atomic data (Ralchenko et al. 2008). A simultaneous fit of elemental abundance, projected rotational velocity and radial velocity was then performed for every identified line using the FITSB2 routine (Napiwotzki et al. 2004). Our analysis pipeline automatically rejects lines that are not present and calculates upper limits for the abundances (see Geier et al., in pre. for details). We detected 37 metal lines of sufficient strength that are not blended by lines of different elements.

We find that the abundances of Balloon 090100001 are normal for an sdB star with similar surface parameters (see Table 2), as the comparison with the well studied short period pulsating sdB Feige $48\left(T_{\text {eff }}=29500 \mathrm{~K}, \log g=5.50\right.$, Heber et al. 2000) shows. Abundances of sdBs with different atmospheric parameters have been derived from optical spectra by Edelmann et al. (1999), Heber et al. (1999) and Napiwotzki et al. (2001). We find for Balloon 090100001 that all metals up to sulfur have subsolar abundances, while iron is consistent with solar abundance. The strongest deviations are present in the carbon and silicon abundances. These elements are known to show

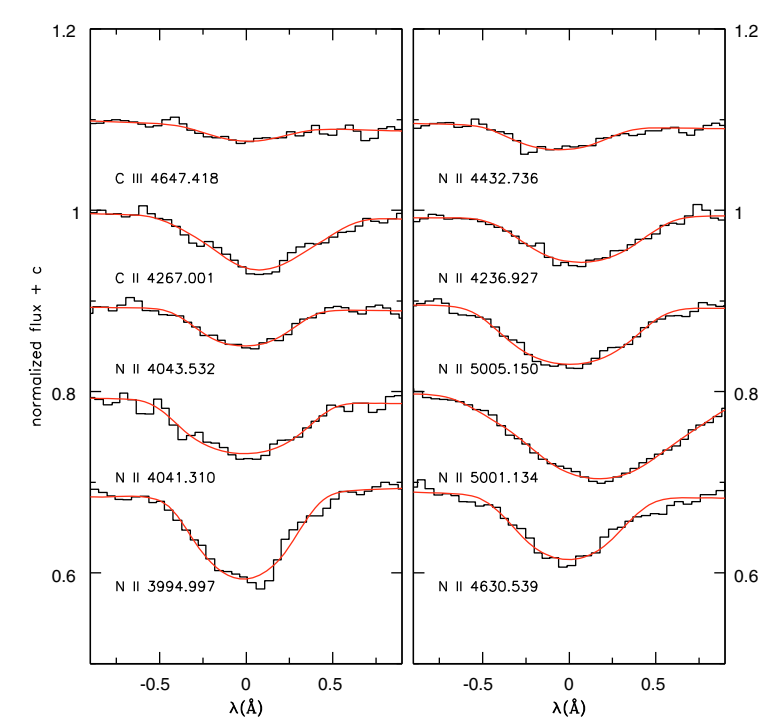

Fig. 3. Fits to the line profiles of a phase-binned spectrum, used for abundance and broadening analysis. The observations are drawn in histogram style.

the largest star-to-star scatter in sdB atmospheres (see Heber \& Edelmann 2004; Edelmann et al. 2006).

This implies that the high pulsation amplitudes are unlikely to be caused by an abundance/metallicity anomaly that is detectable at the surface. Although we used average parameters for $T_{\text {eff }}$ and $\log g$ for all pulsational phases, the measured abundances do not change significantly with pulsational phase.

Our results are fully consistent with the abundance analysis of short-period pulsators using UV spectra obtained with HST/STIS (O'Toole \& Heber 2006). No significant differences in surface abundance have been found between pulsating and non-pulsating sdBs. The same is true for the long-period pulsators analysed so far using UV spectra obtained with the FUSE satellite (Blanchette et al. 2008).

Our spectra have high enough resolution to resolve the line profiles (see Fig. 3). We find that the metal lines of Balloon 090100001 are much broader than in low-amplitude pulsators (Heber et al. 2000) or non-pulsating sdB stars, for which Geier et al. (in prep.) find an average equatorial surfacerotation speed of $V_{\mathrm{e}}=8 \mathrm{~km} \mathrm{~s}^{-1}$. For the phase-binned spectra the line broadening, parametrised as Gaussian broadening $\sigma_{\mathrm{G}}$, varies around $23 \mathrm{~km} \mathrm{~s}^{-1}$ with $\sim 2.5 \mathrm{~km} \mathrm{~s}^{-1}$ difference between the extreme values during the pulsation. The broadening of the average spectrum (all 842 Aug. 2006 spectra combined) amounts to $\sigma_{\mathrm{G}}=27.6 \mathrm{~km} \mathrm{~s}^{-1}$. Parametrised as rotational broadening we get $28.6 \pm 1.0 \mathrm{~km} \mathrm{~s}^{-1}$. Hence we find clear evidence of pulsational broadening of the lines, which implies that also in other largeamplitude pulsating sdB stars the metal-line broadening may have a significant pulsational component, which was already pointed out by Kuassivi et al. (2005) in the case of PG 1605+072 (Heber et al. 1999).

\section{Cross correlation to obtain high-S/N line profiles}

Although the phase-binned spectra have relatively high $S / N \sim 50$, the noise in the individual line profiles is still too high to attempt our mode-identification technique. Therefore we combined the information contained in many narrow line profiles (see Figs. 1 and 2), in order to obtain a single high- $S / N$ profile for each pulsation phase. 
Table 3. Number of narrow spectral lines used for the cross-correlation analysis.

\begin{tabular}{ccccccccc}
\hline \hline C II & C III & FeIII & HeI & MgII & N II & O II & SilII & Total \\
\hline 1 & 1 & 14 & 1 & 1 & 16 & 17 & 5 & 56 \\
\hline
\end{tabular}

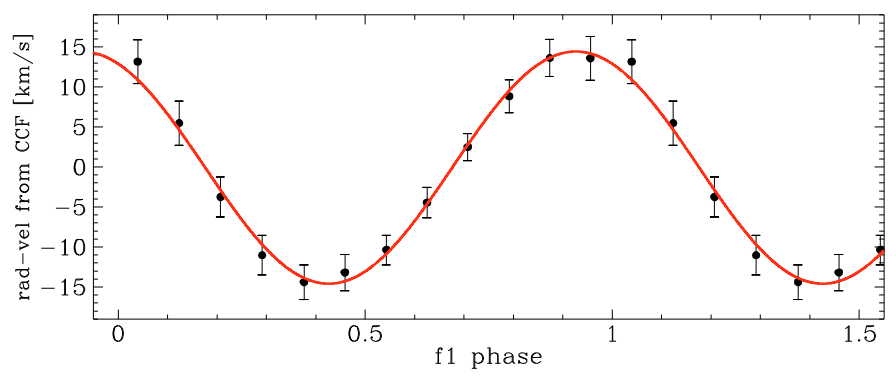

Fig. 4. Main pulsation mode radial-velocity variation as obtained from the cross-correlation analysis.

We identified 56 narrow unblended lines (Table 3), and used their central wavelengths to create a template spectrum consisting of delta functions convolved with the instrumental broadening $\left(5 \mathrm{~km} \mathrm{~s}^{-1}\right.$ Gaussian $\left.\sigma\right)$. The minimal distance between lines selected for the template was kept at $1.5 \AA$, to avoid blends in the cross-correlation function.

For each phase bin we computed the cross-correlation function $(\mathrm{CCF})$ of the stellar spectrum with the template spectrum, using the standard IRAF cross-correlation routine FXCOR. This effectively combined all information contained in 56 separate spectral lines into one CCF profile. The 12 resulting CCF profiles were re-normalized to unity using a 6th-order Legendre polynomial, and have very good $S / N \sim 220$ with a line depth of about 0.15 continuum units. These CCF profiles were used as if they were real line profiles in the mode identification (see Fig. 5, and next section).

To obtain the radial-velocity amplitude of the main pulsation mode in Balloon 090100001 we have fitted a sine curve to the center values of the CCF of the phase-binned spectra. In Fig. 4 we show this fit, with error bars on the CCF centers as reported by the IRAF FXCOR task. We find a radial-velocity amplitude of $14.5 \pm 0.9 \mathrm{~km} \mathrm{~s}^{-1}$, although the error on this amplitude decreases to $0.5 \mathrm{~km} \mathrm{~s}^{-1}$ when we scale the IRAF errors to achieve a reduced $\chi^{2}=1.0$ for the sine fit. This radial-velocity amplitude is significantly lower than that was found by Telting \& Østensen (2006): data from Aug./Sep. 2004 show 18-19 km s${ }^{-1}$ radialvelocity amplitude for the main mode. This significant amplitude reduction of the main mode of Balloon 090100001 over a twoyear period has also been observed photometrically by Baran et al. (2008).

Note that the exposure time (30 s) and the phase binning together give rise to a smearing of the pulsational signal with an effective exposure time of $\sim 45 \mathrm{~s}$. This means that for the main mode the observed amplitudes are suppressed by $2.8 \%$ and for the first harmonic the amplitudes are suppressed by $10.4 \%$ due to smearing.

\section{Identification of the Main Pulsation Mode in Balloon 090100001}

For the mode-identification of the main mode in Balloon 090100001 we used the model of Schrijvers \& Telting (1999) to compute pulsational line-profile variations. In most cases the pulsational variability of metal lines is dominated by the pulsational surface velocity pattern. For the simple fits we present below, we therefore only accounted for the pulsational velocity field, and neglected the effects that local surface-gravity and temperature (i.e. local brightness and equivalent width) variations may have on the metal lines.

We have fitted the $12 \mathrm{CCF}$ profiles with a model of a single spheroidal p-mode, with a ratio of horizontal to vertical surface displacements $k=0.05$, which is adequate given the observed frequencies and estimates for radius and mass of the star. In the modelling we used a linear limb-darkening law with coefficient $\alpha=0.25$. Another parameter of the model is the projected equatorial velocity. A typical value of the equatorial surface-rotation speed of single sdB stars is $V_{\mathrm{e}}=8 \mathrm{~km} \mathrm{~s}^{-1}$ (Geier et al., in prep.). However, in the case of Balloon 090100001 the observed multiplet splitting (Baran et al. 2008) together with the radius estimate of $R=0.24 R_{\odot}\left(\right.$ for $\log g=5.39$ and assumed $M=0.5 M_{\odot}$ ) lead to an expected value of $V_{\mathrm{e}} \sin (i) \sim 1.5-2 \mathrm{~km} \mathrm{~s}^{-1}$. Hence we fixed $V_{\mathrm{e}} \sin (i)=2 \mathrm{~km} \mathrm{~s}^{-1}$, but we have checked that for a rotational velocity as high as $V_{\mathrm{e}} \sin (i)=8 \mathrm{~km} \mathrm{~s}^{-1}$ we get very similar results for the profile fits we describe below.

The 5 free parameters in the fit were the central profile depth, an offset in radial velocity with respect to zero, the pulsation phase, the intrinsic line-broadening $\sigma_{\mathrm{G}}$ (assumed Gaussian), and the physical (i.e. unnormalised) pulsation amplitude $V_{\mathrm{p}}$.

For all $(\ell, m)$ modes with $\ell \leq 2$ we fitted the model for different inclination angles ranging from $i=10^{\circ}$ to $i=80^{\circ}$ in steps of ten degrees. For the radial mode we only computed fits for $i=10^{\circ}$ and $i=70^{\circ}$, and expectedly found identical results, as for this mode the profile variations are independent of the inclination angle. Fits were optimised by minimising the reduced- $\chi^{2}$ value, with the determined $S / N=220$ (see Sect. 4) as error on the intensity value of each individual velocity bin of the CCF spectra. For each best fitting model we computed the expected amplitude of variation of the centroid radial-velocity $M_{1}$ which can be compared to the observed radial-velocity amplitude.

The results of the fits are listed in Table 4 . We list the models for a range of the reduced- $\chi^{2}$ value $\left[\chi_{\min }^{2}, \chi_{\min }^{2}+1\right]$, which happens to correspond to the models that predict the radial-velocity amplitude within $1 \sigma$; the observed radial-velocity amplitude is $14.5 \pm 0.5 \mathrm{~km} \mathrm{~s}^{-1}$ (see Sect. 4). Additionally we list the best fits for every possible $(\ell=2,|m| \leq \ell)$ mode.

We have overplotted the model fit for a radial mode and for the best-fit $\ell=1$ and best-fit $\ell=2$ model onto the CCFs as a function of pulsation phase in Fig. 5, where the mean CCF profile is plotted at the top in the panels. This figure shows that the line profiles modelled for the radial and $\ell=1$ modes describe the observed CCFs well, whereas the profiles expected for nonradial modes with $\ell=2$ do not fit the data properly.

From Table 4 we conclude that modes with $\ell<2$ describe the profile variations much better than modes with $\ell=2$. Based on the reduced- $\chi^{2}$ values the $\ell=2$ modes can be excluded as possible origin of the line-profile variation of the main mode in Balloon 090100001. Additionally, from the rightmost column of Table 4 we find that the $\ell=2$ modes are not able to produce the observed radial-velocity amplitude, for the pulsation amplitude that fits the spectra best. For all modes with $\ell>2$ we expect even worse results: bad profile fits and inability to produce the observed radial-velocity amplitude for realistic pulsation amplitudes. Therefore we conclude, based on simple fits to our highresolution time-resolved data set alone, that the main pulsation mode in Balloon 090100001 is either a radial $(\ell=0)$ or a dipole mode $(\ell=1)$. 


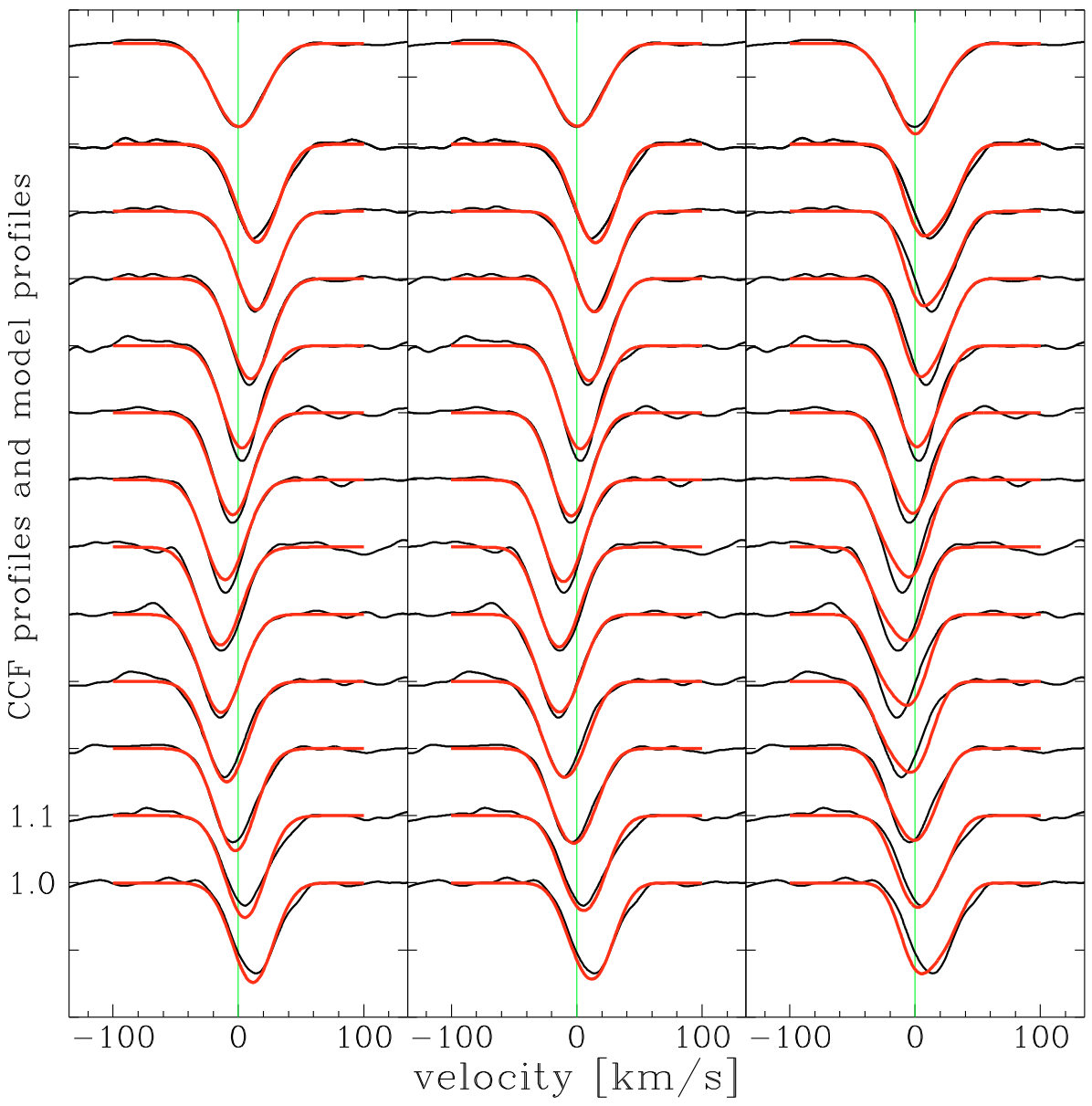

Fig. 5. Observed cross-correlation functions (CCFs), offset as a function of pulsation phase by 0.1 continuum units (phase is increasing upwards), with the mean of all CCFs at the top. In the left panel we have overplotted model profiles for a radial pulsation mode: $\ell=0, i=70^{\circ}$, surface velocity amplitude $22 \mathrm{~km} \mathrm{~s}^{-1}$. In the middle panel we overplot the profile variations expected for a non-radial pulsation with $\ell=1$, $m=-1, i=50^{\circ}$, surface velocity amplitude $35 \mathrm{~km} \mathrm{~s}^{-1}$. In the right panel we overplot the profile variations expected for a non-radial pulsation with $\ell=2, m=-1, i=40^{\circ}$, surface velocity amplitude $38 \mathrm{~km} \mathrm{~s}^{-1}$, which is the best-fit $\ell=2$ mode. Both the radial and $\ell=1$ mode fit better than any $\ell=2$ mode.
Table 4. Results of fits to the CCF profile variations. Listed are the best fits, with reduced $\chi^{2}$ within $\left[\chi_{\min }^{2}, \chi_{\min }^{2}+1\right]$, and below the separation the best fits for every $(\ell=2,|m| \leq \ell)$ combination. Inclination angle is given in degrees, the velocity amplitudes $V_{\mathrm{p}}$ and $A_{\mathrm{M}_{1}}$ in $\mathrm{km} \mathrm{s}^{-1}$, and the line-broadening $\sigma_{\mathrm{G}}$ in $\mathrm{km} \mathrm{s}^{-1}$.

\begin{tabular}{ccccccc}
\hline \hline$\ell$ & $m$ & $i$ & $V_{\mathrm{p}}$ & $\sigma_{\mathrm{G}}$ & $\chi^{2}$ & $A_{\mathrm{M}_{1}}$ \\
\hline 1 & -1 & 50 & $35.1 \pm 0.1$ & $18.06 \pm 0.07$ & 1.62 & 14.59 \\
1 & -1 & 60 & $31.3 \pm 0.1$ & $19.31 \pm 0.06$ & 1.62 & 14.69 \\
1 & -1 & 70 & $28.8 \pm 0.1$ & $19.96 \pm 0.05$ & 1.64 & 14.71 \\
1 & -1 & 80 & $27.5 \pm 0.1$ & $20.28 \pm 0.05$ & 1.65 & 14.71 \\
1 & -1 & 40 & $40.3 \pm 0.1$ & $16.05 \pm 0.08$ & 1.79 & 14.06 \\
0 & 0 & any & $21.5 \pm 0.1$ & $22.60 \pm 0.04$ & 1.91 & 14.66 \\
1 & 0 & 10 & $27.3 \pm 0.1$ & $21.94 \pm 0.04$ & 2.00 & 14.58 \\
1 & 0 & 20 & $28.5 \pm 0.1$ & $21.84 \pm 0.04$ & 2.02 & 14.55 \\
1 & 0 & 30 & $30.8 \pm 0.1$ & $21.64 \pm 0.05$ & 2.09 & 14.47 \\
1 & 0 & 40 & $34.4 \pm 0.1$ & $21.32 \pm 0.05$ & 2.25 & 14.29 \\
1 & 1 & 80 & $27.2 \pm 0.1$ & $20.57 \pm 0.05$ & 2.31 & 14.55 \\
1 & 1 & 70 & $28.5 \pm 0.1$ & $20.28 \pm 0.05$ & 2.34 & 14.53 \\
1 & 1 & 60 & $30.8 \pm 0.1$ & $19.70 \pm 0.06$ & 2.40 & 14.48 \\
1 & 1 & 50 & $34.5 \pm 0.1$ & $18.63 \pm 0.06$ & 2.53 & 14.34 \\
& & & & & & \\
2 & -1 & 40 & $37.6 \pm 0.1$ & $14.74 \pm 0.10$ & 4.20 & 12.09 \\
2 & 0 & 10 & $40.1 \pm 0.2$ & $20.43 \pm 0.05$ & 4.75 & 12.49 \\
2 & 1 & 50 & $38.0 \pm 0.1$ & $16.27 \pm 0.09$ & 5.12 & 12.19 \\
2 & -2 & 80 & $35.1 \pm 0.1$ & $14.40 \pm 0.10$ & 5.27 & 11.09 \\
2 & 2 & 80 & $34.7 \pm 0.1$ & $15.08 \pm 0.11$ & 6.53 & 10.98 \\
\hline
\end{tabular}

The fact that we find marginally better $\chi^{2}$ values for the ( $\ell=1, m=-1$ ) mode than for the radial mode may be due to the fact that we have not accounted for pulsational temperature effects when modeling the line profiles. Although the inclusion of temperature effects in our model will not make any of the $\ell \geq 2$ modes fit the data well, the temperature effects may affect the mode identification for modes that have marginally different $\chi^{2}$ values, such as the radial and dipole modes in Table 4 . In a forthcoming study we intend to investigate these effects in more detail, and we will investigate whether it is possible to use our data set to put modal constraints on the lower-amplitude pulsations in this star.

\section{Summary and conclusions}

We obtained time-resolved high-resolution spectroscopy of the sdBV Balloon $090100001\left(B_{\mathrm{mag}} \sim 11.8\right.$, main period $\left.=356 \mathrm{~s}\right)$ on the $2.6 \mathrm{~m}$ Nordic Optical Telescope with the FIES spectrograph in order to derive pulsational characteristics of, and the atmospheric response to, the dominant pulsational mode in this star. After folding to pulsational phase, the combined spectra have sufficient $S / N$ for detailed analysis.

The abundances of Balloon 090100001 were found to be normal for a star of its kind. Hence the high pulsation amplitudes are unlikely to be caused by an abundance/metallicity anomaly. As expected, the abundances do not vary with pulsational phase of the main mode.

The main-mode radial-velocity amplitude in Aug. 2006 was $14.5 \mathrm{~km} \mathrm{~s}^{-1}$, which is significantly lower than what was observed in Aug./Sep. 2004. A reduction in the main-mode amplitude was also observed photometrically by Baran et al. (2008). 
The broadening of the line profiles of Balloon 090100001 depends on pulsational phase, and the lines are in general much broader than for non-pulsating single sdB stars. Besides having classical broadening, the absorption lines in this sdB star, and possibly in other sdBs as well, are pulsationally broadened.

We used cross-correlation to combine the pulsational information content of 56 carefully selected spectral lines into a single CCF, for each phase-binned spectrum. From simple model fits (only 3D velocity field) to the pulsational line-profile variability in the CCFs, we can exclude a possible $\ell=2$ origin of the main mode in Balloon. Pulsation modes of $\ell \geq 3$ require unrealistic surface amplitudes and were hence not considered.

The line-profile variability of the main mode of Balloon 090100001 is well described by a model of a radial mode, but at this point $\ell=1$ modes cannot be excluded. We intend to present further detailed modeling, including pulsational surface-temperature effects, of the line-profile variability in our high-resolution data set of Balloon 090100001, to differentiate between an $\ell=0$ (i.e. radial) or $\ell=1$ origin of the main mode. We will investigate whether it is possible to use our data set to put modal constraints on the lower-amplitude pulsations in this star. This will require an in-depth study on the importance of pulsational surface variations of the main mode on the diagnostic value of the line-profile variations of the smaller-amplitude modes in this star.

It is comforting to find that the application of our independent mode-identification technique gives results that agree very well with mode identifications based on other (mostly photometric) observations, such as presented by Baran et al. (2008) and Charpinet et al. (2008). The good correspondence between these results strengthens the suggestion that in asteroseismological analyses much weight should be given to observationally derived mode identifications.

Balloon 090100001 is one of the brightest sdB pulsators, and is one of the few targets one could choose for time-resolved high-resolution spectroscopy on 2-4 m-class telescopes. It is natural to expect that better-accuracy data sets might be obtained at $8 \mathrm{~m}$-class telescopes, provided that the instrumental setup allows for time-resolved high-resolution spectroscopy with a cadence of about 10-30 s. Unfortunately, such fast instrumental setups are not typically part of the first-generation instruments on these larger telescopes, implying large overheads between successive observations. Once faster detectors are available for high-resolution spectroscopy on large telescopes, the mode-identification technique discussed in this paper may be applicable to more high-amplitude pulsating sdB stars.

Acknowledgements. Based on observations made with the Nordic Optical Telescope, operated on the island of La Palma jointly by Denmark, Finland, Iceland, Norway, and Sweden, in the Spanish Observatorio del Roque de los Muchachos of the Instituto de Astrofisica de Canarias.

S.G. is supported by the Deutsche Forschungsgemeinschaft through grant He1356/40-4.

R.Ø. is supported by the Research Council of the Universty of Leuven and by the FP6 Coordination Action HELAS of the EU.

\section{References}

Baran, A., Oreiro, R., Pigulski, A., Pérez Hernández, F., \& Ulla, A. 2006, Baltic Astron., 15, 227

Baran, A., Pigulski, A., \& O'Toole, S. J. 2008, MNRAS, 385, 255

Blanchette, J.-P., Chayer, P., Wesemael, F., et al. 2008, ApJ, 678, 1329

Briquet, M., \& Aerts, C. 2003, A\&A, 398, 687

Charpinet, S., Fontaine, G., Brassard, P., \& Dorman, B. 1996, ApJ, 471, L106

Charpinet, S., Fontaine, G., Brassard, P., et al. 1997, ApJ, 483, L123

Charpinet, S., Fontaine, G., Brassard, P., et al. 2005, A\&A, 443, 251

Charpinet, S., Fontaine, G., Brassard, P., et al. 2008, in Hot Subdwarf Stars and Related Objects, ed. U., Heber, C. S., Jeffery, \& R., Napiwotzki, ASPCS, 392, 297

Dorman, B., O’Connell, R. W., \& Rood, R. T. 1993, ApJ, 419, 596

D’Cruz, N. L., Dorman, B., Rood, R. T., \& O'Connell, R. W. 1996, ApJ, 466, 359

Edelmann, H., Heber, U., Napiwotzki, R., Reid, I. N., \& Saffer, R. A. 1999, ASPCS, 169, 546

Edelmann, H., Heber, U., \& Napiwotzki, R. 2006, Baltic Astron., 15, 103

Fontaine, G., Green, E. M., Brassard, P., et al. 2004, Ap\&SS, 291, 379

Green, E. M., Fontaine, G., Reed, M. D., et al. 2003, ApJ, 583, L31

Grevesse, N., \& Sauval, A. J. 1998, SSRv, 85, 161

Van Grootel, V., Charpinet, S., Fontaine, G., et al. 2008, A\&A, 488, 685

Han, Z., Podsiadlowski, P., Maxted, P. F. L., Marsh, T. R., \& Ivanova, N. 2002, MNRAS, 336, 448

Han, Z., Podsiadlowski, P., Maxted, P. F. L., \& Marsh, T. R. 2003, MNRAS, 341, 669

Heber, U. 1986, A\&A, 155, 33

Heber, U., \& Edelmann, H. 2004, Ap\&SS, 291, 341

Heber, U., Reid, I. N., \& Werner, K. 1999, A\&A, 348, L25

Heber, U., Reid, I. N., \& Werner, K. 2000, A\&A, 363, 198

Hu, H., Dupret, M.-A., Aerts, C., et al. 2008, A\&A, 490, 243

Jeffery, C. S., \& Pollacco, D. 2000, MNRAS, 318, 974

Jeffery, C. S., Dhillon ,V. S., Marsh, T. R., \& Ramachandran, B. 2004, MNRAS, 352,699

Kilkenny, D., Koen, C., O’Donoghue, D., \& Stobie, R. S. 1997, MNRAS, 285, 640

Kuassivi, B. A., \& Ferlet, R. 2005, A\&A, 442, 1015

Kurucz, R. L 1992, in Proc. of the 149th Symposium of the IAU, ed. B., Barbuy, \& A., Renzini (Dordrecht: Kluwer Academic Publishers), 225

Lemke, M. 1997, A\&AS, 122, 285

Napiwotzki, R., Edelmann, H., Heber, U., et al. 2001, A\&A, 378, L17

Napiwotzki, R., Yungelson, L., Nelemans, G., et al. 2004, ASPCS, 318, 402

Oreiro, R., Ulla, A., Pérez Hernández, F., et al. 2004, A\&A, 418, 243

Oreiro, R., Pérez Hernández, F., Ulla, A., et al. 2005, A\&A, 438, 257

O'Toole, S. J., \& Heber, U. 2006, A\&A, 452, 579

O'Toole, S. J., Bedding, T. R., Kjeldsen, H., et al. 2000, ApJ, 537, L53

O'Toole, S. J., Jørgensen, M. S., Kjeldsen, H., et al. 2003, MNRAS, 340, 856

Østensen, R. H., Telting, J. H., Heber, U., \& Jeffery, C. S. 2008, in Hot Subdwarf Stars and Related Objects, ed. U., Heber, C. S., Jeffery, \& R., Napiwotzki, ASPCS, 392, 301

Ralchenko, Yu., \& Kramida, A. E. 2008, Reader J. and NIST ASD Team http://physics.nist.gov/PhysRefData/ASD/index.html\#Team

Randall, S. K., Fontaine, G., Charpinet, S., et al. 2006, ApJ, 648, 637

Saffer R. A., Bergeron P., Koester D., \& Liebert J. 1994, ApJ, 432, 351

Schrijvers C., \& Telting J. H. 1999, A\&A, 342, 453

Telting J. H, \& Schrijvers C. 1997, A\&A, 317, 723

Telting J. H., \& Østensen R. H. 2004, A\&A, 419, 685

Telting J. H., \& Østensen R .H. 2006, A\&A, 450, 1149

Telting J. H., Østensen R. H., Geier S., et al. 2008, in Hot Subdwarf Stars and Related Objects, ed. U., Heber, C. S., Jeffery, \& R., Napiwotzki, ASPCS, 392, 265

Vučković M., Aerts C., Østensen R. H., et al. 2007, A\&A, 471, 605

Woolf, V. M., Jeffery, C. S., \& Pollacco, D. L. 2002, MNRAS, 329, 497 